\title{
Mesenchymal stem cells regulate maintenance of neural stem cells through VEGFR2 and Notch signaling pathways
}

\author{
Zhifeng Deng ${ }^{1}$, Zhumin Liu ${ }^{1}$, Wei Tu ${ }^{1}$,Yang Wang ${ }^{2}$, Yuanlei Lou ${ }^{2}$ \\ ${ }^{I}$ Department of Neurosurgery, the Second Affiliated Hospital of Nanchang University; ${ }^{2}$ Institute of Urology, Nanchang University, \\ Nanchang 330006, China
}

Mesenchymal stem cells (MSCs) transplantation is known to be potential to improve functional outcome after stroke. In our previous study, we demonstrated that MSCs could regulate the maintenance of neural stem cells (NSCs) either in vitro or in vivo. However, the precise mechanism is still unclear. MSCs can produce a variety of cytokines and growth factors including vascular endothelial growth factor (VEGF). It has been shown that the proliferation and differentiation of NSCs may be influenced by Notch signaling pathway and VEGF stimulation. In this study, we investigated that MSCs stimulated the self-renewal and differentiation of NSCs regulated by Notch signaling pathway and VEGFR 2 . NSCs dissociated from embryonic mouse cerebral cortex (E12) were cocultured with MSCs or Human Microvascular Endothelial Cells (HMVEC). To confirm the effect of VEGFR 2 , SU5416 (a selective inhibitor of the tyrosine kinase activity of the VEGFR 2 ) was applied in coculture system. For control, NSCs were cocultured with NIH3T3. RT-PCR results showed that expression of Notch1 and Hes1 genes in NSCs up-regulated by MSCs and HMVEC, whereas the expression of Mash1 down-regulated. Conversely, the expression level of Notch1 and Hes1 genes in NSCs was significantly lower when VEGFR2 was blocked or NSCs were cocultured with NIH3T3, and the expression of Mash1 was at high level. Determination of nestin (a marker of NSCs) and GFAP (a marker of mature astrocyte) showed that MSCs and HMVEC promoted the expression of nestin and inhibited the expression of GFAP in NSCs. Our results demonstrate that MSCs promote NSCs proliferation and inhibit its differentiation through the VEGFR2 present on NSCs. Moreover, we provide the evidence that VEGF signals transmitted by the VEGFR may $^{2}$ activate Notch signaling, which regulate different responses of progenitor cells. In conclusion, the data indicate that MSCs act via VEGFR 2 and Notch signaling pathway, either directly or indirectly, to regulate maintenance of NSCs. Keywords: mesenchymal stem cells, neural stem cells, VEGFR2, Notch Cell Research (2008) 18:s59. doi: 10.1038/cr.2008.149; published online 4 August 2008

Correspondence: Yang Wang

E-mail: wangy63cn@sina.com

Supported by National Natural Science Foundation of China (No.

30560156); Natural Science Foundation of Jiangxi province (No. 0540087) 